Mathematical Modelling and Analysis

Volume 21 Number 4, July 2016, 550-568

http://dx.doi.org/10.3846/13926292.2015.1186756

(c) Vilnius Gediminas Technical University, 2016
Publisher: Taylor\&Francis and VGTU

http://www.tandfonline.com/TMMA

ISSN: $1392-6292$

eISSN: $1648-3510$

\title{
A Mathematical Study on the System of Partial Differential Equations Describing Reverse-Flow Reactor
}

\author{
Ratno Bagus Edy Wibowo ${ }^{a}$, Takashi Suzuki ${ }^{b}$ and \\ Ryo Takahashi ${ }^{c}$ \\ ${ }^{a}$ Department of Mathematics, Faculty of Mathematics and Natural Sciences, \\ Brawijaya University \\ JL. Veteran, 65145 Malang, Indonesia \\ ${ }^{b}$ Division of Mathematical Science, Department of Systems Innovation, \\ Graduate School of Engineering Science, Osaka University \\ Machikaneyamacho 1-3, 560-8531 Toyonakashi, Japan \\ ${ }^{c}$ Mathematics Education, Faculty of Education, Nara University of Education \\ Takabatake-cho, 630-8528 Nara-shi, Japan \\ E-mail(corresp.): rbagus@ub.ac.id \\ E-mail: suzuki@sigmath.es.osaka-u.ac.jp \\ E-mail: r-takaha@nara-edu.ac.jp
}

Received November 28, 2015; revised May 1, 2016; published online July 1, 2016

\begin{abstract}
The RFR (reverse flow reactor) has been widely studied for abatement of lean methane emission from coal mining or natural gas piping system. We show a global-in-time existence of the solution to a nonlinear system of partial differential equations modeling RFR.
\end{abstract}

Keywords: reverse-flow reactor, methane gas, switching time, compartment, parabolichyperbolic system.

AMS Subject Classification: 35JXX; 35KXX; 35lXX; 35F50; 35F55; 35F60; 35F61.

\section{Introduction}

The purpose of the present paper is to study a mathematical model concerning Reverse Flow Reactor (referred to as RFR) used for catalytic oxidation of lean methane emission. Here, the main concept of RFR is a flow, whose directions are periodically changing through the packed bed reactor. It has been widely studied for the abatement of lean methane emissions from coal mining or natural gas piping systems, see [8]. In fact, such emissions contribute to global warming potential, which is considerably dangerous for the environment, and therefore, it is indispensable to treat this emission into a harmless compound. 
The use of the reverse flow principle as a transient operation procedure for catalytic reactors may be hopeful, under a dynamic interaction between microscaled catalysts and macroscaled reactor to produce more favorable concentration and temperature profiles in the catalytic process, see [4]. Hence, studies on RFR are proposed actively in chemical engineering process, due to its importance in applications to destruct pollutants, see [6].

Several mathematical models have been proposed to predict the behavior of the reverse-flow reaction in terms of temperature and concentration. One of the simplest model is concerned with a single reaction without changing the number of moles of reactants, see $[3,9,15]$, that is,

$$
\left\{\begin{array}{cl}
\left(\varepsilon_{g} \rho_{g} c_{g}+\varepsilon_{s} \rho_{s} c_{s}\right) \frac{\partial \Theta}{\partial t}= & \\
\lambda_{e f} \frac{\partial^{2} \Theta}{\partial x^{2}}-\rho_{g}^{o} c_{g} U(t) \frac{\partial \Theta}{\partial x}+(-\delta H) W_{1}(X, \Theta), & \text { in }(0, L) \times(0, T), \\
\varepsilon_{g} \rho_{g} \frac{\partial X}{\partial t}=-U(t) \rho_{g}^{o} \frac{\partial X}{\partial x}+W_{2}(X, \Theta), & \text { in }(0, L) \times(0, T),
\end{array}\right.
$$

where $\Theta=\Theta(x, t)$ and $X=X(x, t), 0 \leq x \leq L, 0 \leq t<T$, denote the temperature and the fractional conversion of the catalyst, respectively. It is thus a model of one-space dimension, with $L$ standing for the bed length. Here, $\varepsilon, \rho$, and $c$ are physical constants with the subscripts $g, s$, and $o$ standing for the states of gas, of solid, and outside the system, respectively. Furthermore, $\lambda_{e f}$ and $\delta H$ are chemical quantities, and the nonlinearities $W_{i}(X, \Theta), i=1,2$, are the rates of chemical reaction. See Appendix for more details of these constants and functions.

The principal flow direction is thus controled by the linear velocity $U(t)=$ $\kappa(t) u$ defined by

$$
\kappa(t)= \begin{cases}+1, & \text { at } t \in\left[2 m \tau^{*},(2 m+1) \tau^{*}\right), \\ -1, & \text { at } t \in\left[(2 m+1) \tau^{*},(2 m+2) \tau^{*}\right) \quad \text { for } m=0,1, \ldots,\end{cases}
$$

where $u$ is a constant and $\tau^{*}$ stands for a half of the cycle duration. Then we provide the initial condition:

$$
\left.\Theta\right|_{t=0}=\Theta_{0},\left.\quad X\right|_{t=0}=X_{0} \quad \text { on }[0, L],
$$

for given functions $\Theta_{0}=\Theta_{0}(x)$ and $X_{0}=X_{0}(x)$, and also the boundary conditions

$$
\begin{array}{ll}
X=\frac{1-\kappa(t)}{2} X, & \lambda_{e f} \frac{\partial \Theta}{\partial x}=\frac{1+\kappa(t)}{2} u c_{g} \rho_{g}^{o}\left(\Theta-\Theta_{i n p}\right) \\
X=\frac{1+\kappa(t)}{2} X, & \lambda_{e f} \frac{\partial \Theta}{\partial x}=-\frac{1-\kappa(t)}{2} u c_{g} \rho_{g}^{o}\left(\Theta-\Theta_{i n p}\right)
\end{array}
$$

at $\{0\} \times(0, T)$ and $\{L\} \times(0, T)$, respectively, for a given constant $\Theta_{i n p}$, where " $X=X$ " or " $\Theta=\Theta$ " is regarded as an empty condition. When RFR works, complex and characteristic phenomena are experimentally-observed, see [14, 15,16] and references therein. They are also observed in numerical simulations with the aid of mathematical models, see [7,10,11,14,15,16,17] and references therein. According to numerical simulations by Budhi et al., complicated and 
sensitive asymptotic behavior of solutions can be observed, see [2]. For this reason, it is important to analyze RFR not only by experiments but also by using mathematical models. The main result of the present paper is to show the unique existence of the global-in-time solution to (1.1)-(1.5). Furthermore, we also study more complicated models which may accompany compartment, see Section 4 for details.

Normalizing physical constants, we thus take the system of parabolic and hyperbolic equations

$$
\begin{aligned}
& \varepsilon \frac{\partial \Theta}{\partial t}=D \frac{\partial^{2} \Theta}{\partial x^{2}}-U(t) \frac{\partial \Theta}{\partial x}+F(X, \Theta), \Theta \geq 0, \\
& \frac{\partial X}{\partial t}+U(t) \frac{\partial X}{\partial x}=G(X, \Theta), \quad \text { in }(0, L) \times(0, T),
\end{aligned}
$$

where $\varepsilon, D>0$ are constants. The initial condition is imposed as

$$
\Theta(x, 0)=\Theta_{0}(x), \quad X(x, 0)=X_{0}(x) \quad \text { on }[0, L],
$$

while the boundary conditions take the form

$$
\begin{aligned}
& D \frac{\partial \Theta}{\partial x}=\frac{1+\kappa(t)}{2} u\left(\Theta-\Theta_{i n p}\right), \quad X=\frac{1-\kappa(t)}{2} X \\
& D \frac{\partial \Theta}{\partial x}=-\frac{1-\kappa(t)}{2} u\left(\Theta-\Theta_{i n p}\right), \quad X=\frac{1+\kappa(t)}{2} X
\end{aligned}
$$

on $\{0\} \times(0, T)$ and $\{L\} \times(0, T)$, respectively. Concerning the nonlinearities, we assume

$$
\begin{aligned}
& F \in C^{1}(\mathbf{R} \times[0, \infty)), \quad G \in C^{1}(\mathbf{R} \times[0, \infty)), \\
& |F(X, \Theta)| \leq M\left(1+|X|^{\gamma_{1}}+\Theta^{\gamma_{2}}\right), \\
& |G(X, \Theta)| \leq M\left(1+|X|+\Theta^{\gamma_{3}}\right), \\
& \gamma_{1}, \gamma_{2}, \gamma_{3}>0, \quad \gamma_{2} \leq 1, \quad \gamma_{1} \gamma_{3} \leq 1,
\end{aligned}
$$

where $M>0$ and $\gamma_{i}(i=1,2,3)$ are constants. This assumption of the nonlinearity is satisfied in most of models, see [3,9,15].

The main theorem is stated under the following notations. First, $\operatorname{Lip}\left(\overline{Q_{T}}\right)$ denotes the set of Lipschitz continuous functions on $\overline{Q_{T}}$ with respect to $(x, t)$, where $Q_{T}=(0, L) \times(0, T)$. Next, $C^{l, \frac{l}{2}}\left(\overline{Q_{T}}\right)$ is the Hölder space, composed of continuous functions on $\overline{Q_{T}}$ together with their all the derivatives of the form $\partial_{t}^{r} \partial_{x}^{s}$ for $2 r+s<l$ and take the finite norm

$$
\|u\|_{\left(Q_{T}\right)}^{(l)}=\langle u\rangle_{Q_{T}}^{(l)}+\sum_{j=0}^{[l]}\langle u\rangle_{Q_{T}}^{(j)},
$$

where $l \in \mathcal{Z}=\{0,1,2, \ldots\}$ and

$$
\begin{aligned}
& \langle u\rangle_{Q_{T}}^{(0)}=\|u\|_{\left(Q_{T}\right)}^{(0)}=\max _{Q_{T}}|u|, \quad\langle u\rangle_{Q_{T}}^{(j)}=\sum_{2 r+s=j}\left\|\partial_{t}^{r} \partial_{x}^{s} u\right\|_{\left(Q_{T}\right)}^{(0)}, \\
& \langle u\rangle_{Q_{T}}^{(l)}=\langle u\rangle_{x, Q_{T}}^{(l)}+\langle u\rangle_{t, Q_{T}}^{\left(\frac{l}{2}\right)}, \quad\langle u\rangle_{x, Q_{T}}^{(l)}=\sum_{2 r+s=[l]}\left\langle\partial_{t}^{r} \partial_{x}^{s} u\right\rangle_{x, Q_{T}}^{(l-[l])},
\end{aligned}
$$




$$
\begin{aligned}
& \langle u\rangle_{t, Q_{T}}^{\left(\frac{l}{2}\right)}=\sum_{0<l-2 r-s<2}\left\langle\partial_{t}^{r} \partial_{x}^{s} u\right\rangle_{t, Q_{T}}^{\left(\frac{l-2 r-s}{2}\right)}, \\
& \langle u\rangle_{x, Q_{T}}^{(\gamma)}=\sup _{(x, t),(y, t) \in \overline{Q_{T}}, x \neq y,|x-y| \leq \rho_{0}} \frac{|u(x, t)-u(y, t)|}{|x-y|^{\gamma}}, \\
& \langle u\rangle_{t, Q_{T}}^{(\gamma)}=\sup _{(x, t),(x, s) \in \overline{Q_{T}}, t \neq s,|t-s| \leq \rho_{0}} \frac{|u(x, t)-u(x, s)|}{|t-s|^{\gamma}},
\end{aligned}
$$

for $0<\gamma<1$. Finally, $W_{q}^{2 l, l}\left(Q_{T}\right), l \in \mathcal{Z}, q \geq 1$, denotes the Banach space consisting of the elements of the Lebesgue space $L^{q}\left(Q_{T}\right)$ having generalized derivatives of the form $\partial_{t}^{r} \partial_{x}^{s}$ with any $r$ and $s$ satisfying the inequality $2 r+s \leq$ $2 l$. Its norm is defined by

$$
\|u\|_{q, Q_{T}}^{(2 l)}=\sum_{j=0}^{2 l}|u|_{q, Q_{T}}^{(j)}
$$

where

$$
|u|_{q, Q_{T}}^{(j)}=\sum_{2 r+s=j}\left|\partial_{t}^{r} \partial_{x}^{s} u\right|_{q, Q_{T}}, \quad|u|_{q, Q_{T}}=\left(\int_{0}^{T} \int_{0}^{L}|u(x, t)|^{q} d x d t\right)^{\frac{1}{q}} .
$$

Let $Q=Q_{\infty}=(0, L) \times(0,+\infty)$ and define $\Gamma_{i}, i=1,2,3$, by the following:

$$
\begin{aligned}
\Gamma_{1}= & \left\{(x, t) \in Q \mid t=k \tau^{*}, k=0,1, \ldots\right\}, \\
\Gamma_{2}= & \left\{(x, t) \in Q \mid t=x / u+2 k \tau^{*}, t \in\left[2 k \tau^{*},(2 k+1) \tau^{*}\right], k=0,1, \ldots\right\} \\
& \cup\left\{(x, t) \mid t=x / u+2 k \tau^{*}-\left(L-\tau^{*}\right), t \in\left[2 k \tau^{*},(2 k+1) \tau^{*}\right],\right. \\
& \quad k=0,1, \ldots\}, \\
\Gamma_{3}= & \left\{(x, t) \in Q \mid t=-x / u+2 k \tau^{*}, t \in\left[(2 k-1) \tau^{*},(2 k) \tau^{*}\right], k=1,2, \ldots\right\} \\
\cup & \left\{(x, t) \in Q \mid t=-x / u+2 k \tau^{*}+\left(L-\tau^{*}\right), t \in\left[(2 k-1) \tau^{*},(2 k) \tau^{*}\right],\right. \\
& k=1,2, \ldots\},
\end{aligned}
$$

for $u \tau^{*} \leq L$, and

$$
\begin{aligned}
\Gamma_{1}= & \left\{(x, t) \in Q \mid t=k \tau^{*}, k=0,1, \ldots\right\}, \\
\Gamma_{2}= & \left\{(x, t) \in Q \mid t=x / u+2 k \tau^{*}, t \in\left[2 k \tau^{*},(2 k+1) \tau^{*}\right], k=0,1, \ldots\right\}, \\
\Gamma_{3}= & \left\{(x, t) \mid t=-x / u+L+(2 k+1) \tau^{*}, t \in\left[(2 k-1) \tau^{*},(2 k) \tau^{*}\right],\right. \\
& \quad k=1,2, \ldots\},
\end{aligned}
$$

for $u \tau^{*}>L$. Then we put $\Gamma=\Gamma_{1} \cup \Gamma_{2} \cup \Gamma_{3}$, and also

$$
\Gamma_{2,0}^{\prime}= \begin{cases}\left\{(x, t) \mid t=x / u-\left(L-\tau^{*}\right), t \in\left[0, \tau^{*}\right]\right\}, & \text { if } u \tau^{*} \leq L, \\ \emptyset, & \text { if } u \tau^{*}>L\end{cases}
$$

Finally, we define $P_{\tau^{*}}$ by

$$
P_{\tau^{*}}=\left\{(x, t) \mid x=0, L, t=m \tau^{*}, m=0,1, \ldots\right\} .
$$


Theorem 1. Given $\Theta_{0} \in C^{\alpha}[0, L]$ and $X_{0} \in C^{1}(0, L] \cap L^{\infty}(0, L)$ satisfying $\alpha \in(0,1), \Theta_{0} \geq 0$, and given $F$ and $G$ satisfying (1.10)-(1.13), there exists a unique solution $(\Theta, X)$ to (1.6)-(1.9) such that

$$
\begin{aligned}
& \Theta \in C^{2,1}(\bar{Q} \backslash \Gamma) \cap W_{q, l o c}^{2,1}\left(Q \backslash P_{\tau^{*}}\right), \\
& X \in C^{1,1}\left((\bar{Q} \backslash \Gamma) \cup \Gamma_{2,0}^{\prime}\right) \cap C\left(\bar{Q} \backslash\left(\Gamma_{2} \cup \Gamma_{3}\right)\right), \\
& \Theta(\cdot, t) \rightarrow \Theta_{0} \quad \text { locally uniformly in }(0, L], \\
& \lim _{t \downarrow(2 m-1) \tau^{*}} \Theta(\cdot, t)=\lim _{t \uparrow(2 m-1) \tau^{*}} \Theta(\cdot, t)
\end{aligned}
$$

locally uniformly in

$$
\begin{cases}{[0, L) \backslash\left\{\left(u \tau^{*},(2 m-1) \tau^{*}\right)\right\},} & \text { if } u \tau^{*} \leq L, \\ {[0, L),} & \text { if } u \tau^{*}>L\end{cases}
$$

and

$$
\lim _{t \downarrow 2 m \tau^{*}} \Theta(\cdot, t)=\lim _{t \uparrow 2 m \tau^{*}} \Theta(\cdot, t)
$$

locally uniformly in

$$
\begin{cases}(0, L] \backslash\left\{\left(L-u \tau^{*}, 2 m \tau^{*}\right)\right\}, & \text { if } u \tau^{*} \leq L, \\ (0, L], & \text { if } u \tau^{*}>L,\end{cases}
$$

for any $q>1$ and for every $m=1,2, \ldots$

This paper is organized as follow. We prepare several auxiliary lemmas in Section 2, and prove Theorem 1 in Section 3. More complicated models of RFR are studied in Section 4. In Appendix, various variables arising in the systems in Sections 1 and 4 are explained. Henceforth, $C_{i}(i=1,2, \ldots)$ denote positive constants whose subscripts are renewed in each section.

\section{Preliminaries}

The first aim of this section is to show the local-in-time existence and uniqueness for the parabolic-hyperbolic system

$$
\begin{cases}\varepsilon \Theta_{t}=D \Theta_{x x}-u \Theta_{x}+F(X, \Theta), \quad \Theta \geq 0, & \text { in } Q_{T}, \\ X_{t}+u X_{x}=G(X, \Theta), & \text { in } Q_{T}, \\ D \Theta_{x}-u \Theta=-u \Theta_{i n p}, \quad X=0, & \text { on }\{0\} \times(0, T), \\ \Theta_{x}=0, & \text { on }\{L\} \times(0, T), \\ \left.\Theta\right|_{t=0}=\Theta_{0},\left.\quad X\right|_{t=0}=X_{0}, & \text { on }[0, L] .\end{cases}
$$

For the purpose, we recall the classical results for the linear parabolic equation

$$
\begin{cases}u_{t}=L_{x t} u+f(x, t), & \text { in } Q_{T}, \\ u_{x}(\xi, t)+\beta(\xi, t) u=g(\xi, t), & \text { in }\{0, L\} \times(0, T), \\ \left.u\right|_{t=0}=u_{0}, & \text { in }(0, L),\end{cases}
$$


where $L_{x t}$ denotes the operator defined by

$$
L_{t x}=a(x, t) \frac{\partial^{2}}{\partial x^{2}}+b(x, t) \frac{\partial}{\partial x}+c(x, t)
$$

and the coefficients and inhomogeneous data satisfy

$$
f, a, b, c \in C^{\alpha}([0, L] \times[0, T)), \quad g(0, \cdot), g(L, \cdot), \beta(0, \cdot), \beta(L, \cdot) \in C[0, T),
$$

for some $\alpha \in(0,1)$.

Lemma 1 [ [5]]. Assume that (2.3) holds and that $u_{0} \in C[0, L]$ satisfies $\left.u\right|_{t=0}=u_{0}$ on $[0, L]$. Then, there exists a unique solution to (2.2) satisfying

$$
\lim _{t \downarrow 0} u(\cdot, t)=u_{0} \quad \text { uniformly on }[0, L] .
$$

Lemma 2 [ [5]]. Assume that (2.3) holds and that $u_{0} \in C(0, L)$. Then, there exists a unique solution to (2.2) satisfying

$$
\lim _{t \downarrow 0} u(\cdot, t)=u_{0} \quad \text { locally uniformly in }(0, L) .
$$

Note that the difference between Lemmas 1 and 2 is only whether the condition $\left.u\right|_{t=0}=u_{0}$ on $[0, L]$ is satisfied or not.

Now we state the result of the local-in-time existence and uniqueness.

Proposition 1. Given $0 \leq \Theta_{0} \in C[0, L]$ and $X_{0} \in C^{1}(0, L] \cap C[0, L]$, and given $F$ and $G$ satisfying (1.10), there exists $T>0$, depending only on $F$, $G,\left\|X_{0}\right\|_{L^{\infty}(0, L)},\left\|\Theta_{0}\right\|_{L^{\infty}(0, L)}, \Theta_{i n p}, \varepsilon, D$ and $u$, such that (2.1) has a unique solution $(\Theta, X)$ satisfying

$$
\begin{aligned}
& \Theta \in C^{2,1}\left(Q_{T}\right) \cap C^{1,0}\left(\overline{Q_{T}} \backslash\{t=0\}\right) \cap C\left(\overline{Q_{T}} \backslash\{(0,0)\}\right), \\
& X \in C^{1,1}\left(\overline{Q_{T}} \backslash\{(x, t) \mid t=u x, t \in[0, T]\}\right), \\
& \Theta(\cdot, t) \rightarrow \Theta_{0} \quad \text { locally uniformly in }(0, L] \text { as } t \downarrow 0 .
\end{aligned}
$$

Proof. For simplicity, we assume that $\varepsilon=D=u=1$. The proof for other cases is similar to that for the case $\varepsilon=D=u=1$.

First, we shall prove the local-in-time existence. Fix $0<T \ll 1$ which is determined later on. Given $\tilde{\Theta} \in \operatorname{Lip}\left(\overline{Q_{T}}\right)$, we can solve

$$
\begin{cases}X_{t}+X_{x}=G(X, \tilde{\Theta}), & \text { in }(0, L) \times(0, T), \\ X=0, & \text { on }\{0\} \times(0, T), \\ \left.X\right|_{t=0}=X_{0}, & \text { on }[0, L],\end{cases}
$$


provided that $T$ is so small. Note that $T$ is determined only by $G,\left\|X_{0}\right\|_{L^{\infty}(0, L)}$ and $\|\tilde{\Theta}\|_{\infty}$. In fact, (2.7) is equivalent to the integral equation $X(x, t)=\left\{\begin{array}{l}X_{0}(z(x, t ; 0))+\int_{0}^{t} \tilde{P}(z(x, t ; s), s) d s, \quad \text { for }(x, t) \in \overline{Q_{T}} \cap\{t \leq x\}, \\ \int_{0}^{x} \tilde{P}(y, w(x, t ; y)) d y, \quad \text { for }(x, t) \in \overline{Q_{T}} \cap\{t>x\},\end{array}\right.$

where $\tilde{P}(x, t)=G(X(x, t), \tilde{\Theta}(x, t))$ and $z(x, t ; s)$ (resp. $w(x, t ; y))$ denotes the characteristic line which begins at $(x-t, 0)$ (resp. $(0, t-x))$ and ends at $(x, t)$ (resp. $(x, t))$, see [1]. Note that

$$
\begin{aligned}
& \frac{d z(x, t ; s)}{d s}=1, \quad \text { for every } s \in[0, t], \\
& \frac{d w(x, t ; y)}{d y}=1, \quad \text { for every } y \in[0, x], \\
& X=X(x, t) \in C^{1}\left(\overline{Q_{T}} \backslash\{t=x\}\right) \cap L^{\infty}\left(Q_{T}\right) .
\end{aligned}
$$

A direct calculation shows

$$
\|X\|_{\infty} \leq\left\|X_{0}\right\|_{L^{\infty}(0, L)}+T \cdot A_{G}\left(\|X\|_{\infty},\|\tilde{\Theta}\|_{\infty}\right),
$$

where

$$
A_{G}\left(K, K^{\prime}\right)=\max _{\xi \in[-K, K], \eta \in\left[-K^{\prime}, K^{\prime}\right]}|G(\xi, \eta)| .
$$

For the unique solution $X=X(x, t)$ to $(2.7)$, we consider

$$
\begin{cases}\Theta_{t}=\Theta_{x x}-\Theta_{x}+F(X, \tilde{\Theta}), & \text { in }(0, L) \times(0, T), \\ \Theta_{x}-\Theta=-\Theta_{i n p}, & \text { on }\{0\} \times(0, T), \\ \Theta_{x}=0, & \text { on }\{L\} \times(0, T), \\ \left.\Theta\right|_{t=0}=\Theta_{0}, & \text { on }[0, L] .\end{cases}
$$

The standard theory for linear parabolic equations guarantees that the problem (2.10) has a unique solution $\Theta=\Theta(x, t)$ satisfying $\Theta \in W_{q}^{2,1}\left(Q_{T}\right)$ for $q \in(3, \infty)$ and $\Theta \in C^{2,1}\left(Q_{T} \backslash\{t=x\}\right)$, see $[12,13]$. Set

$$
\begin{aligned}
& \bar{\Theta}=\bar{\Theta}(x, t)=\max \left\{\left\|\Theta_{0}\right\|_{L^{\infty}(0, L)}, \Theta_{i n p}\right\}+t\|F(X, \tilde{\Theta})\|_{\infty}, \\
& \underline{\Theta}=\underline{\Theta}(x, t)=-t\|F(X, \tilde{\Theta})\|_{\infty}, \quad \bar{\Theta}_{*}=\bar{\Theta}-\Theta, \quad \underline{\Theta}_{*}=\underline{\Theta}-\Theta .
\end{aligned}
$$

Then, it holds that

$$
\left\{\begin{array}{l}
\left(\bar{\Theta}_{*}\right)_{t} \geq\left(\bar{\Theta}_{*}\right)_{x x}-\left(\bar{\Theta}_{*}\right)_{x}, \quad \text { in }(0, L) \times(0, T), \\
\left(\bar{\Theta}_{*}\right)_{x}-\bar{\Theta}_{*} \leq 0, \quad \text { on }\{0\} \times(0, T) \\
\left(\bar{\Theta}_{*}\right)_{x}=0, \quad \text { on }\{L\} \times(0, T) \\
\bar{\Theta}_{*}(x, 0) \geq 0, \quad \text { for } x \in[0, L]
\end{array}\right.
$$

and that

$$
\left\{\begin{array}{l}
\left(\underline{\Theta}_{*}\right)_{t} \leq\left(\underline{\Theta}_{*}\right)_{x x}-\left(\underline{\Theta}_{*}\right)_{x}, \quad \text { in }(0, L) \times(0, T), \\
\left(\underline{\Theta}_{*}\right)_{x}-\underline{\Theta}_{*} \geq 0, \quad \text { on }\{0\} \times(0, T), \\
\left(\underline{\Theta}_{*}\right)_{x}=0, \quad \text { on }\{L\} \times(0, T), \\
\underline{\Theta}_{*}(x, 0), \leq 0 \text { for } x \in[0, L],
\end{array}\right.
$$


which implies

$$
\underline{\Theta} \leq \Theta \leq \bar{\Theta} \quad \text { in }[0, L] \times[0, T) .
$$

Hence we obtain

$$
\|\Theta\|_{\infty} \leq \max \left\{\left\|\Theta_{0}\right\|_{L^{\infty}(0, L)}, \Theta_{i n p}\right\}+T \cdot A_{F}\left(\|\tilde{X}\|_{\infty},\|\tilde{\Theta}\|_{\infty}\right),
$$

where

$$
A_{F}\left(K, K^{\prime}\right)=\max _{\xi \in[-K, K], \eta \in\left[-K^{\prime}, K^{\prime}\right]}|F(\xi, \eta)| .
$$

Now we introduce the set

$$
\mathcal{S}_{T}=\left\{\Theta \in L^{\infty}\left(Q_{T}\right) \mid\|\Theta\|_{\infty} \leq K_{0}\right\}
$$

and the mapping

$$
\Phi(\tilde{\Theta})=\Theta \quad \text { for } \tilde{\Theta} \in \mathcal{S}_{T}
$$

where

$$
K_{0}=1+\max \left\{\left\|\Theta_{0}\right\|_{L^{\infty}(0, L)}, \Theta_{i n p}\right\}
$$

and $\Theta=\Theta(x, t)$ is a unique solution to (2.10) via (2.7). From the inequalities (2.9) and (2.11), we see that $\Phi$ maps $\mathcal{S}_{T}$ to itself if $0<T \ll 1$. Note that $T$ depends only on $F, G,\left\|X_{0}\right\|_{L^{\infty}(0, L)},\left\|\Theta_{0}\right\|_{L^{\infty}(0, L)}$ and $\Theta_{i n p}$ (for the other cases, besides on $\varepsilon, D$ and $u$ ).

We now claim that $\Phi$ is a contraction map on $\mathcal{S}_{T}$ if $0<T \ll 1$ is small enough. Given $\tilde{\Theta}_{1}, \tilde{\Theta}_{2} \in \mathcal{S}_{T}$, the function $\widehat{\Theta}=\Theta_{1}-\Theta_{2}=\Phi\left(\tilde{\Theta}_{1}\right)-\Phi\left(\tilde{\Theta}_{2}\right)$ satisfies

$$
\begin{cases}\widehat{\Theta}_{t}=\widehat{\Theta}_{x x}-\widehat{\Theta}_{x}+\left(F\left(\tilde{X}_{1}, \tilde{\Theta}_{1}\right)-F\left(\tilde{X}_{2}, \tilde{\Theta}_{2}\right)\right), & \text { in }(0, L) \times(0, T), \\ \widehat{\Theta}_{x}-\widehat{\Theta}=0, & \text { on }\{0\} \times(0, T), \\ \widehat{\Theta}_{x}=0, & \text { on }\{L\} \times(0, T), \\ \widehat{\Theta}(x, 0)=0, & \text { on }[0, L],\end{cases}
$$

where $\tilde{X}_{i}(i=1,2)$ is a unique solution to $(2.7)$ for $\tilde{\Theta}=\tilde{\Theta}_{i}$. Set

$$
L_{0}=1+\left\|X_{0}\right\|_{L^{\infty}(0, L)} .
$$

Since $G=G(\xi, \eta)$ satisfies (1.10) and since $\tilde{\Theta}_{i} \in \mathcal{S}_{T}$, the inequality (2.9) implies that $\left\|\tilde{X}_{i}\right\|_{\infty} \leq L_{0}$ if $T$ is small enough. Comparing $\widehat{\Theta}=\widehat{\Theta}(x, t)$ with the function

$$
\pm t \cdot A_{F}^{\prime}\left(L_{0}, K_{0}\right) \cdot\left(\left\|\tilde{X}_{1}-\tilde{X}_{2}\right\|_{\infty}+\left\|\tilde{\Theta}_{1}-\tilde{\Theta}_{2}\right\|_{\infty}\right)
$$

admits the estimate

$$
\|\widehat{\Theta}\|_{\infty} \leq T \cdot A_{F}^{\prime}\left(L_{0}, K_{0}\right) \cdot\left(\left\|\tilde{X}_{1}-\tilde{X}_{2}\right\|_{\infty}+\left\|\tilde{\Theta}_{1}-\tilde{\Theta}_{2}\right\|_{\infty}\right),
$$

where

$$
A_{F}^{\prime}\left(K, K^{\prime}\right)=\max _{\xi \in[-K, K], \eta \in\left[-K^{\prime}, K^{\prime}\right]}|\nabla F(\xi, \eta)|
$$


We have, on the other hand,

$$
\tilde{X}_{1}(x, t)-\tilde{X}_{2}(x, t)=\left\{\begin{array}{r}
\int_{0}^{t} \tilde{P}_{1}(z(x, t ; s), s)-\tilde{P}_{2}(z(x, t ; s), s) d s, \\
\text { for }(x, t) \in \overline{Q_{T}} \cap\{t \leq x\}, \\
\int_{0}^{x} \tilde{P}_{1}(y, w(x, t ; y))-\tilde{P}_{2}(y, w(x, t ; y)) d y, \\
\text { for }(x, t) \in \overline{Q_{T}} \cap\{t>x\},
\end{array}\right.
$$

by (2.8), where

$$
\tilde{P}_{i}(x, t)=G\left(\tilde{X}_{i}(x, t), \tilde{\Theta}_{i}(x, t)\right), \quad \text { for } i=1,2 .
$$

Then it holds that

$$
\left\|\tilde{X}_{1}-\tilde{X}_{2}\right\|_{\infty} \leq T \cdot A_{G}^{\prime}\left(L_{0}, K_{0}\right) \cdot\left(\left\|\tilde{X}_{1}-\tilde{X}_{2}\right\|_{\infty}+\left\|\tilde{\Theta}_{1}-\tilde{\Theta}_{2}\right\|_{\infty}\right),
$$

so that

$$
\left\|\tilde{X}_{1}-\tilde{X}_{2}\right\|_{\infty} \leq 2 T \cdot A_{G}^{\prime}\left(L_{0}, K_{0}\right) \cdot\left\|\tilde{\Theta}_{1}-\tilde{\Theta}_{2}\right\|_{\infty},
$$

if $T$ is small enough, where

$$
A_{G}^{\prime}\left(K, K^{\prime}\right)=\max _{\xi \in[-K, K], \eta \in\left[-K^{\prime}, K^{\prime}\right]}|\nabla G(\xi, \eta)| .
$$

Consequently, inequalities (2.13) and (2.14) yield

$$
\|\widehat{\Theta}\|_{\infty} \leq \frac{1}{2}\left\|\tilde{\Theta}_{1}-\tilde{\Theta}_{2}\right\|_{\infty},
$$

for some $0<T \ll 1$ depending only on $F, G,\left\|X_{0}\right\|_{L^{\infty}(0, L)},\left\|\Theta_{0}\right\|_{L^{\infty}(0, L)}$ and $\Theta_{\text {inp }}$ (for the other cases, besides on $\varepsilon, D$ and $u$ ), which implies that $\Phi$ is a contraction map on $\mathcal{S}_{T}$.

At this stage, we perform the iteration scheme by setting $\Theta^{(0)}=\Theta_{0}$ and $\Theta^{(j+1)}=\Phi\left(\Theta^{(j)}\right)(j=0,1, \ldots)$. Note that the iteration scheme works well in this setting. Then we find that the limit function $\Theta=\lim _{j \rightarrow \infty} \Theta^{(j)}$ is a solution to

$$
\begin{cases}\Theta_{t}=\Theta_{x x}-\Theta_{x}+F(X, \Theta), & \text { in }(0, L) \times(0, T), \\ \Theta_{x}-\Theta=-\Theta_{i n p}, & \text { on }\{0\} \times(0, T), \\ \Theta_{x}=0, & \text { on }\{L\} \times(0, T), \\ \left.\Theta\right|_{t=0}=\Theta_{0}, & \text { on }[0, L]\end{cases}
$$

and that $\Theta=\Theta(x, t)$ satisfies (2.4) and (2.6) by the parabolic regularities (see $[12,13]$ ) and Lemmas $1-2$, where $X=\lim _{j \rightarrow \infty} X^{(j)}$ and $X^{(j)}$ is a unique solution to (2.7) for $\tilde{\Theta}=\Theta^{(j)}$. Note that this $X=X(x, t)$ is well-defined and a solution to (2.7) for $\tilde{\Theta}=\Theta$ satisfying (2.5) by (2.8) and (2.14). Hence, the local-in-time existence is proven.

Next, we shall prove the non-negativities of $\Theta=\Theta(x, t)$ constructed above. Let $(X, \Theta)$ be the solution constructed above. We introduce the function

$$
\Theta^{(\mu)}=\Theta^{(\mu)}(x, t)=e^{-\mu t} \Theta(x, t), \quad \mu>0
$$


and obtain

$$
\Theta_{t}^{(\mu)}=\Theta_{x x}^{(\mu)}-\Theta_{x}^{(\mu)}-(\mu-c) \Theta^{(\mu)}, \quad \text { in }(0, L) \times(0, T),
$$

where $c=c(x, t)$ is a bounded function. For $\mu \gg 1$, it holds that $\mu-c \geq 0$ in $(0, L) \times(0, T)$. Assume that $\mu \gg 1$ and $\min \frac{}{Q_{T}} \Theta^{(\mu)}<0$. Then, $\min \frac{}{Q_{T}} \Theta^{(\mu)}$ is attained at some point on the parabolic boundary by the maximum principle and the non-negativity of the initial data $\Theta_{0}$. However, $\min \frac{\bar{Q}_{T}}{} \Theta^{(\mu)}<0$ fails because of the boundary condition of (2.1) and Hopf's lemma, and hence $\min _{\overline{Q_{T}}} \Theta^{(\mu)} \geq 0$, namely $\min \overline{Q_{T}} \Theta \geq 0$.

Finally, we shall prove the uniqueness. Let $\left(X_{i}, \Theta_{i}\right)(i=1,2)$ be a solution to (2.1) satisfying (2.4)-(2.6), and put $\tilde{\Theta}=\Theta_{1}-\Theta_{2}, \tilde{X}=X_{1}-X_{2}$. Then, it holds that

$$
\left\{\begin{array}{l}
\tilde{\Theta}_{t}=\tilde{\Theta}_{x x}-\tilde{\Theta}_{x}+\left(F\left(X_{1}, \Theta_{1}\right)-F\left(X_{2}, \Theta_{2}\right)\right), \quad \text { in }(0, L) \times(0, T), \\
\tilde{X}_{t}+\tilde{X}_{x}=G\left(X_{1}, \Theta_{1}\right)-G\left(X_{2}, \Theta_{2}\right), \quad \text { in }(0, L) \times(0, T), \\
\tilde{\Theta}_{x}-\tilde{\Theta}=0, \quad \tilde{X}=0, \quad \text { on } \quad\{0\} \times(0, T), \\
\tilde{\Theta}_{x}=0, \quad \text { on } \quad\{0\} \times(0, T), \\
\tilde{\Theta}(x, 0)=\tilde{X}(x, 0)=0, \quad \text { for } x \in[0, L] .
\end{array}\right.
$$

Multiplying the first equation of (2.18) by $\tilde{\Theta}$ gives

$$
\begin{aligned}
& \frac{1}{2} \frac{d}{d t} \int_{0}^{L} \tilde{\Theta}^{2} d x=\left[\tilde{\Theta} \tilde{\Theta}_{x}\right]_{x=0}^{x=L}-\frac{1}{2}\left[\tilde{\Theta}^{2}\right]_{x=0}^{x=L}-\int_{0}^{L} \tilde{\Theta}_{x}^{2} d x \\
& \quad+\int_{0}^{L} \tilde{\Theta}\left(F\left(X_{1}, \Theta_{1}\right)-F\left(X_{2}, \Theta_{2}\right)\right) d x \\
& \leq C\left(F,\left\|X_{1}\right\|_{\infty},\left\|X_{2}\right\|_{\infty},\left\|\Theta_{1}\right\|_{\infty},\left\|\Theta_{2}\right\|_{\infty}\right) \int_{0}^{L} \tilde{\Theta}(|\tilde{X}|+|\tilde{\Theta}|) d x \\
& \leq C\left(F,\left\|X_{1}\right\|_{\infty},\left\|X_{2}\right\|_{\infty},\left\|\Theta_{1}\right\|_{\infty},\left\|\Theta_{2}\right\|_{\infty}\right) \int_{0}^{L}\left(\frac{1}{2} \tilde{X}^{2}+\frac{3}{2} \tilde{\Theta}^{2}\right) d x
\end{aligned}
$$

Similarly, multiplying the second equation of (2.18) by the $\tilde{X}$ gives

$$
\begin{aligned}
& \frac{1}{2} \frac{d}{d t} \int_{0}^{L} \tilde{X}^{2} d x=-\frac{1}{2}\left[\tilde{X}^{2}\right]_{x=0}^{x=L}+\int_{0}^{L} \tilde{X}\left(G\left(X_{1}, \Theta_{1}\right)-G\left(X_{2}, \Theta_{2}\right)\right) d x \\
& \leq C\left(G,\left\|X_{1}\right\|_{\infty},\left\|X_{2}\right\|_{\infty},\left\|\Theta_{1}\right\|_{\infty},\left\|\Theta_{2}\right\|_{\infty}\right) \int_{0}^{L} \tilde{X}(|\tilde{X}|+|\tilde{\Theta}|) d x \\
& \leq C\left(G,\left\|X_{1}\right\|_{\infty},\left\|X_{2}\right\|_{\infty},\left\|\Theta_{1}\right\|_{\infty},\left\|\Theta_{2}\right\|_{\infty}\right) \int_{0}^{L}\left(\frac{3}{2} \tilde{X}^{2}+\frac{1}{2} \tilde{\Theta}^{2}\right) d x
\end{aligned}
$$

Consequently we obtain

$$
\frac{d}{d t} \int_{0}^{L}\left(\tilde{X}^{2}+\tilde{\Theta}^{2}\right) d x \leq C\left(F, G,\left\|X_{1}\right\|_{\infty},\left\|X_{2}\right\|_{\infty},\left\|\Theta_{1}\right\|_{\infty},\left\|\Theta_{2}\right\|_{\infty}\right) \int_{0}^{L}\left(\tilde{X}^{2}+\tilde{\Theta}^{2}\right) d x
$$

and conclude $\tilde{X}=\tilde{\Theta}=0$ on $\overline{Q_{T}}$ by using the Gronwall's inequality and the initial condition $\tilde{\Theta}(x, 0)=\tilde{X}(x, 0)=0$ for $x \in[0, L]$. The proof is complete. 
Proposition 2. Assume that $F$ and $G$ satisfy (1.10), and that if $u \tau^{*} \leq L$, $0 \leq \Theta_{0} \in C(0, L) \cap L^{\infty}(0, L)$ and $X_{0} \in C^{1}\left((0, L] \backslash\left\{L-u \tau^{*}\right\}\right) \cap L^{\infty}(0, L)$, otherwise, $0 \leq \Theta_{0} \in C(0, L) \cap L^{\infty}(0, L)$ and $X_{0} \in C^{1}((0, L]) \cap L^{\infty}(0, L)$. Then, there exists $T>0$, depending only on $F, G,\left\|X_{0}\right\|_{L^{\infty}(0, L)},\left\|\Theta_{0}\right\|_{L^{\infty}(0, L)}, \Theta_{\text {inp }}$, $\varepsilon, D$ and $u$, such that (2.1) has a unique solution $(\Theta, X)$ satisfying

$$
\begin{aligned}
& \Theta \in C^{2,1}((0, L) \times(0, T)) \cap C^{1,0}\left(\overline{Q_{T}} \backslash\{t=0\}\right) \cap C\left(\overline{Q_{T}} \backslash\{(0,0),(L, 0)\}\right), \\
& X \in C^{1,1}\left(\overline{Q_{T}} \backslash\{(x, t) \mid t=u x, t \in[0, T]\}\right), \\
& \lim _{t \downarrow 0} \Theta(\cdot, t)=\Theta_{0} \quad \text { locally uniformly in } \begin{cases}(0, L] \backslash\left\{L-u \tau^{*}\right\}, & \text { if } u \tau^{*} \leq L, \\
(0, L], & \text { if } u \tau^{*}>L .\end{cases}
\end{aligned}
$$

The proof of Proposition 2 is similar to that of Proposition 1, and so we omit it here.

The second aim of this section is to provide two elementary results used to show Theorem 1.

Lemma 3. Assume that $\varphi=\varphi(t) \in C_{a b s}[0, T], f=f(t) \in C[0, T], \gamma>0$ and

$$
\varphi^{\prime} \leq \gamma(1+\varphi+f) \quad \text { in }(0, T],
$$

for some constant $\gamma>0$. Then it holds that

$$
\varphi(t) \leq-1+\gamma e^{\gamma t}\left(\int_{0}^{t} e^{-\gamma \tau} f(\tau) d \tau+\frac{1+\varphi(0)}{\gamma}\right), \quad \text { for all } t \in[0, T] .
$$

Lemma 3 is shown by solving the standard diffrential inequality, and so it is omitted here.

Lemma 4. Assume that $\varphi=\varphi(t) \in C_{a b s}[0, T]$ and

$$
\varphi^{\prime} \leq k_{1}+k_{2} \varphi+k_{3} \int_{0}^{t} e^{-k_{4} \tau} \varphi(\tau) d \tau \quad \text { in }(0, T]
$$

for some constants $k_{i}>0(i=1,2,3,4)$. Then, there exists $g=g(t) \in C[0, T]$, determined by $\varphi(0)$ and $k_{1}, \ldots, k_{4}$, such that

$$
\varphi(t) \leq e^{k_{2} t}\left(\varphi(0)+k_{1} t+k_{3} \int_{0}^{t} g(\tau) d \tau\right), \quad \text { for all } t \in[0, T] .
$$

Proof. We put

$$
\xi=\xi(t)=\int_{0}^{t} e^{-k_{4} \tau} \varphi(\tau) d \tau
$$


Then it follows from (2.22) that

$$
\left(\xi^{\prime}(t)+k_{4} \xi(t)\right)^{\prime} \leq k_{1} e^{-k_{4} t}+K\left(\xi^{\prime}(t)+k_{4} \xi(t)\right),
$$

where $^{\prime}=\frac{d}{d t}$ and $K=\max \left\{1, k_{2}, k_{3} / k_{4}\right\}$. We solve (2.24) to find

$$
\xi(t) \leq e^{-k_{4} t} \int_{0}^{t} e^{\left(k_{4}+K\right) \tau}\left\{\frac{k_{1}}{k_{4}+K}\left(1-e^{-\left(k_{4}+K\right) \tau}\right)+\varphi(0)\right\} d \tau=: g(t),
$$

which means

$$
\varphi^{\prime} \leq k_{1}+k_{2} \varphi+k_{3} g(t)
$$

We solve this differential inequality and conclude (2.23).

\section{Proof of the Theorem 1}

First, we note that the problem (1.6)-(1.9) has the symmetry. In fact, for every $m \in\{0,2,4, \ldots\}$ (resp. $m \in\{1,3,5, \ldots\},),(1.6)-(1.9)$ on $\left[2 m \tau^{*},(2 m+1) \tau^{*}\right)$ ) (resp. $\left.\left[(2 m+1) \tau^{*},(2 m+2) \tau^{*}\right)\right)$ is reduced to the problem (2.1) replaced $Q_{T}$ by $Q_{\tau^{*}}$, by change of variables $(x, t) \mapsto\left(x, t-2 m \tau^{*}\right)(\operatorname{resp} . \quad(x, t) \mapsto$ $\left.\left(-x, t-(2 m+1) \tau^{*}\right)\right)$. By combining this fact with Propositions $1-2$ and their proofs, and by regarding $\lim _{t \uparrow m \tau^{*}}(\Theta(\cdot, t), X(\cdot, t))$ as the initial data at time $t=m \tau^{*}, m=1,2, \ldots$, we find that Theorem 1 holds if it is shown that for the solution $\Theta=\Theta(x, t)$ to $(2.1)$ for $T=\tau^{*}$, there exists $C_{1}>0$ such that

$$
\|\Theta\|_{\infty} \leq C_{1}
$$

where $C_{1}$ depends on $\tau^{*}$ and given data $\left\|X_{0}\right\|_{L^{\infty}(0, L)},\left\|\Theta_{0}\right\|_{L^{\infty}(0, L)}, \Theta_{\text {inp }}, M$, $\gamma_{1}, \gamma_{2}, \gamma_{3}, \varepsilon, D$ and $u$, and is bounded as long as they are bounded. Actually, we see from (3.1) that the solution $(\Theta, X)$ to (1.6)-(1.9), satisfying (1.14)-(1.18), extends in time, step by step, as $T=\tau^{*}, T=2 \tau^{*}, \ldots$.

In the remainder of this section, we assume that $\varepsilon=D=u=1$ because the proof for other cases is similar to that for the case $\varepsilon=D=u=1$. To derive the $L^{\infty}$-bound (3.1), we use the energy method to obtain the following differential inequality.

Lemma 5. Assume that $(\Theta, X)$ be a solution to (2.1) satisfing the properties (2.4)-(2.6) or (2.19)-(2.21). Then, there exist positive constants $C_{2}=$ $C_{2}(L, M)$ and $C_{3}=C_{3}(L, M)$ such that

$$
\begin{aligned}
& \frac{d}{d t}\|\psi(t)\|_{2}^{2}-\frac{4 p}{p+1}\|\psi(t)\|_{2}^{2} \leq \Theta_{i n p}^{p+1}-\frac{4 p}{p+1}\|\psi(t)\|_{H^{1}}^{2} \\
& +(p+1) C_{2}\left(\|\psi(t)\|_{\frac{2\left(p+\gamma_{2}\right)}{p+1}}^{\frac{2\left(p+\gamma_{2}\right)}{p+1}}+\|\psi(t)\|_{\frac{2 p s}{p+1}}^{\frac{2 p s}{p+1}}\right) \\
& +(p+1) C_{2}\left\{\int_{0}^{t} e^{-\gamma_{1} s^{\prime} C_{3} \tau}\|\psi(\tau)\|_{\frac{2 \gamma_{1} \gamma_{3} s^{\prime}}{p+1}}^{\frac{2 \gamma_{1} \gamma_{3} s^{\prime}}{p+1}} d \tau+\frac{1+\left\|X_{0}\right\|_{\gamma_{1} s^{\prime}}^{\gamma_{1} s^{\prime}}}{\gamma_{1} s^{\prime} C_{3}}\right\}
\end{aligned}
$$

for $t \in\left(0, \tau^{*}\right), p>1$ and $s, s^{\prime} \in(1, \infty)$ with $1 / s+1 / s^{\prime}=1$, where

$$
\psi(x, t)=\Theta^{\frac{p+1}{2}}(x, t) .
$$


Proof. Multiplying the parabolic equation of (2.1) by $\Theta^{p}, p \geq 1$, and integrating on $[0, L]$ in $x$, we have

$$
\frac{1}{p+1} \frac{d}{d t} \int_{0}^{L} \Theta^{p+1} d x=I+I I
$$

where

$$
I=I(t)=\int_{0}^{L} \Theta^{p} \Theta_{x x}-\Theta^{p} \Theta_{x} d x, \quad I I=I I(t)=\int_{0}^{L} \Theta^{p} F(\Theta, X) d x .
$$

The term $I$ is estimated by

$$
\begin{aligned}
I & =\left[\Theta^{p} \Theta_{x}\right]_{x=0}^{x=L}-p \int_{0}^{L} \Theta^{p-1} \Theta_{x}^{2} d x-\frac{1}{p+1} \int_{0}^{L}\left(\Theta^{p+1}\right)_{x} d x \\
& \leq-\frac{4 p}{(p+1)^{2}} \int_{0}^{L}\left(\Theta^{\frac{p+1}{2}}\right)_{x}^{2} d x+\left.\left\{\Theta^{p}\left(\Theta_{i n p}-\Theta\right)+\frac{1}{p+1} \Theta^{p+1}\right\}\right|_{x=0} \\
& \leq \frac{\Theta_{i n p}^{p+1}}{p+1}-\frac{4 p}{(p+1)^{2}}\left\|\psi_{x}(t)\right\|_{2}^{2}
\end{aligned}
$$

recall (3.3), where we have used the non-negativity of $\Theta$ and the boundary condition. The term $(p+1) \times I I$ is estimated by

$$
\begin{aligned}
(p+1) \times I I & =(p+1) \int_{0}^{L} \psi^{\frac{2 p}{p+1}} F\left(\psi^{\frac{2}{p+1}}, X\right) d x \\
& \leq(p+1) M \int_{0}^{L} \psi^{\frac{2 p}{p+1}}\left(1+|X|^{\gamma_{1}}+\psi^{\frac{2 \gamma_{2}}{p+1}}\right) d x \\
& \leq(p+1) C_{4}(L, M)\left(1+\|\psi\|_{\frac{2\left(p+\gamma_{2}\right)}{p+1}}^{\frac{2\left(p+\gamma_{2}\right)}{p+1}}+\|\psi\|_{\frac{2 p s}{p+1}}^{\frac{2 p s}{p+1}}+\|X\|_{\gamma_{1} s^{\prime}}^{\gamma_{1} s^{\prime}}\right)
\end{aligned}
$$

for all $s, s^{\prime} \in(1, \infty)$ satisfying $1 / s+1 / s^{\prime}=1$. Consequently, it follows that

$$
\begin{aligned}
& \frac{d}{d t}\|\psi(t)\|_{2}^{2}-\frac{4 p}{p+1}\|\psi(t)\|_{2}^{2} \leq \Theta_{i n p}^{p+1}-\frac{4 p}{p+1}\|\psi(t)\|_{H^{1}}^{2} \\
& +(p+1) C_{4}(L, M)\left(1+\|\psi(t)\|_{\frac{2\left(p+\gamma_{2}\right)}{p+1}}^{\frac{2\left(p+\gamma_{2}\right)}{p+1}}+\|\psi(t)\|_{\frac{2 p s}{p+1}}^{\frac{2 p s}{p+1}}+\|X(t)\|_{\gamma_{1} s^{\prime}}^{\gamma_{1} s^{\prime}}\right)
\end{aligned}
$$

for all $t \in(0, T), p \geq 1$ and $s, s^{\prime} \in(1, \infty)$ satisfying $1 / s+1 / s^{\prime}=1$.

In turn, we multiply the hyperbolic equation of $(2.1)$ by $X^{q-1} X, q \geq 1$, so that

$$
\frac{1}{q+1} \frac{d}{d t} \int_{0}^{L}|X|^{q+1} d x+\frac{1}{q+1} \int_{0}^{L}\left(|X|^{q+1}\right)_{x} d x=\int_{0}^{L}|X|^{q-1} X G(X, \Theta) d x
$$

or

$$
\frac{d}{d t} \int_{0}^{L} X^{q+1} d x \leq(q+1) \int_{0}^{L}|X|^{q-1} X G(X, \Theta) d x
$$


recall that $X=0$ at $\{0\} \times(0, L)$. The right-hand side is estimated by

$$
\begin{aligned}
(q & +1) \int_{0}^{L}|X|^{q-1} X G(X, \Theta) d x \\
& \leq(q+1) M \int_{0}^{L}|X|^{q}\left(1+|X|+\psi \frac{2 \gamma_{3}}{p+1}\right) d x \\
& \leq(q+1) C_{5}(L, M)\left\{1+\int_{0}^{L}|X|^{q+1} d x+\|\psi\|_{\frac{2 \gamma_{3}(q+1)}{p+1}}^{\frac{2 \gamma_{3}(q+1)}{p+1}}\right\},
\end{aligned}
$$

by the assumption (1.12). Thus we obtain

$$
\frac{d}{d t} \int_{0}^{L}|X|^{q+1} d x \leq(q+1) C_{5}(L, M)\left\{1+\int_{0}^{L}|X|^{q+1} d x+\|\psi\|_{\frac{2 \gamma_{3}(q+1)}{p+1}}^{\frac{2 \gamma_{3}(q+1)}{p+1}}\right\},
$$

for all $t \in(0, T)$ and $q \geq 1$. Applying Lemma 3 to (3.5), we get

$$
\begin{aligned}
\|X(t)\|_{q+1}^{q+1} \leq- & +(q+1) C_{0} e^{(q+1) C_{5} t} \\
& \times\left\{\int_{0}^{t} e^{-(q+1) C_{0} \tau} \phi_{p, q}(\tau) d \tau+\frac{1+\|X(0)\|_{q+1}^{q+1}}{(q+1) C_{5}}\right\},
\end{aligned}
$$

for all $t \in(0, T), p \geq 1$ and $q \geq 1$, where

$$
\phi_{p, q}(s)=\|\psi(s)\|_{\frac{2 \gamma_{3}(q+1)}{p+1}}^{\frac{2 \gamma_{3}(q+1)}{p+1}} .
$$

Finally, we organize (3.4) and (3.6) for $q+1=\gamma_{1}+s^{\prime}$, and conclude the desired inequality (3.2).

Proof. Proof of Theorem 1: As already stated above, we have only to show (3.1). We put $s=(p+1) / p$ and $s^{\prime}=p+1$ in (3.2) and see from Lemma 5 and (1.13) that

$$
\begin{aligned}
\phi^{\prime} \leq & -\frac{4 p}{p+1}\|\psi(t)\|_{H^{1}}^{2}+\left\{\Theta_{i n p}^{p+1}+\frac{C_{2}}{\gamma_{1} C_{3}}\left(1+\left\|X_{0}\right\|_{\gamma_{1}(p+1)}^{\gamma_{1}(p+1)}\right)\right\} \\
& +(p+1) C_{6}(L, M) \phi+(p+1) C_{7}(L, M) \int_{0}^{t} e^{-\gamma_{1}(p+1) C_{3} \tau} \varphi(\tau) d \tau
\end{aligned}
$$

for $t \in\left(0, \tau^{*}\right)$ and $p>1$ by the assumption (1.13), where

$$
\phi=\phi(t)=\|\psi(t)\|_{2}^{2}=\|\Theta(t)\|_{p+1}^{p+1} .
$$

Applying Lemma 4 for (3.7) and noting the form of $g(t)$ defined by (2.25) show

$$
\|\Theta(t)\|_{p+1} \leq C_{8}
$$

for some $C_{8}=C_{8}\left(\tau^{*},\left\|X_{0}\right\|_{L^{\infty}(0, L)},\left\|\Theta_{0}\right\|_{L^{\infty}(0, L)}, \Theta_{i n p}, M, \gamma_{1}, \gamma_{2}, \gamma_{3}, \varepsilon, D, u\right)>0$ independent of $p$ and for all $p>1$. Consequently, taking the limit $p \rightarrow \infty$ assures the desired estimate (3.1). 


\section{Concluding Remarks}

In this section, we discuss more complicated models. The models which we study are the following $(\mathrm{A})-(\mathrm{C})$.

(A) ODE-Hyperbolic-Hyperbolic system

$$
\left\{\begin{array}{l}
\left\{\varepsilon_{s} c_{s} \rho_{s}+(1-\varepsilon) \varepsilon_{p} c_{g} \rho_{g}\right\} \frac{\partial \Theta_{s}}{\partial t}=\alpha_{0} \alpha_{v}\left(\Theta_{g}-\Theta_{s}\right)+(-\delta H) W_{1}\left(X, \Theta_{s}\right), \\
\varepsilon c_{g} \rho_{g} \frac{\partial \Theta_{g}}{\partial t}=-U(t) c_{g} \rho_{g}^{o} \frac{\partial \Theta_{g}}{\partial x}-\alpha_{0} \alpha_{v}\left(\Theta_{g}-\Theta_{s}\right), \\
\varepsilon_{g} \frac{\partial X}{\partial t}=-U(t) \rho_{g}^{o} \frac{\partial X}{\partial x}+W_{2}\left(X, \Theta_{s}\right),
\end{array}\right.
$$

with the initial-boundary conditions

$$
\begin{array}{lrl}
\left.\Theta_{s}\right|_{t=0}=\Theta_{s, 0},\left.\quad \Theta_{g}\right|_{t=0}=\Theta_{g, 0},\left.\quad X\right|_{t=0}=X_{0}, & & \text { on }[0, L], \\
\Theta_{g}=\frac{1-\kappa(t)}{2} \Theta_{g}, \quad X=\frac{1-\kappa(t)}{2} X, & \text { at }\{0\} \times(0, T), \\
\Theta_{g}=\frac{1+\kappa(t)}{2} \Theta_{g}, \quad X=\frac{1-\kappa(t)}{2} X, & \text { at }\{L\} \times(0, T),
\end{array}
$$

for given functions $\Theta_{s, 0}=\Theta_{s, 0}(x), \Theta_{g, 0}=\Theta_{s, 0}(x)$ and $X_{0}=X_{0}(x)$, where $\kappa(t)$ is as in (1.2) and " $\Theta_{g}=\Theta_{g}$ " or " $X=X$ " is regarded as an empty condition. Note that there is no boundary condition on $\Theta_{s}=\Theta_{s}(x, t)$. The model (4.1)(4.4) was used for studies of RFOs for exothermic and endothermic processes, see [7] and [9], respectively.

(B) Parabolic-Hyperbolic-ODE-Hyperbolic system

$$
\begin{aligned}
& \left\{\begin{array}{l}
\left\{\varepsilon_{s} c_{s} \rho_{s}+(1-\varepsilon) \varepsilon_{p} c_{g} \rho_{g}\right\} \frac{\partial \Theta_{s}}{\partial t} \\
\quad=\lambda_{s, e f} \frac{\partial^{2} \Theta_{s}}{\partial x^{2}}+\alpha_{0} \alpha_{v}\left(\Theta_{g}-\Theta_{s}\right)+\eta(-\delta H) W_{1}\left(X_{s}, \Theta_{s}\right), \\
\varepsilon c_{g} \rho_{g} \frac{\partial \Theta_{g}}{\partial t}=-U(t) c_{g} \rho_{g}^{o} \frac{\partial \Theta_{g}}{\partial x}-\alpha_{0} \alpha_{v}\left(\Theta_{g}-\Theta_{s}\right), \\
\varepsilon_{p}(1-\varepsilon) \rho_{g} \frac{\partial X_{s}}{\partial t}=\beta_{0} a_{v} \rho_{g}\left(X_{g}-X_{s}\right)+\eta W_{2}\left(X_{s}, \Theta_{s}\right), \\
\varepsilon \frac{\partial X_{g}}{\partial t}=-U(t) \rho_{g}^{o} \frac{\partial X_{g}}{\partial x}-\beta_{0} a_{v} \rho_{g}\left(X_{g}-X_{s}\right), \quad \text { in }(0, L) \times(0, T),
\end{array}\right. \\
& \left.\Theta_{s}\right|_{t=0}=\Theta_{s, 0},\left.\quad \Theta_{g}\right|_{t=0}=\Theta_{g, 0}, \\
& \left.X_{s}\right|_{t=0}=X_{s, 0},\left.\quad X_{g}\right|_{t=0}=X_{g, 0}, \quad \text { on }[0, L] \text {, } \\
& \frac{\partial \Theta_{s}}{\partial x}=0, \quad \Theta_{g}=\frac{1-\kappa(t)}{2} \Theta_{g}, \quad X_{g}=\frac{1-\kappa(t)}{2} X_{g}, \quad \text { at }\{0\} \times(0, T) \text {, } \\
& \frac{\partial \Theta_{s}}{\partial x}=0, \quad \Theta_{g}=\frac{1+\kappa(t)}{2} \Theta_{g}, \quad X_{g}=\frac{1-\kappa(t)}{2} X_{g}, \quad \text { at }\{L\} \times(0, T) \text {, }
\end{aligned}
$$

for given functions $\Theta_{s, 0}=\Theta_{s, 0}(x), \Theta_{g, 0}=\Theta_{s, 0}(x)$ and $X_{0}=X_{0}(x)$, where $\kappa(t)$ is as in (1.2) and " $\Theta_{g}=\Theta_{g}$ " or " $X=X$ " is regarded as an empty condition. Note that there is no boundary condition on $X_{s}=X_{s}(x, t)$. The model (4.5)(4.8) describes a two-phase model accounting for longitudinal shear dispersion in a solid phase and mass transfer limitations inside and outside the catalyst particles under the condition that temperature gradients catalyst are not high, 
and was studied experimentally and numerically in [11].

(C) System with compartment

RFR devices with compartment are often used in applications, see [16] for details. One of how to formulate them is as follows. Assume that there are $N$-rooms in the packed bed whose length is $L$, for instance, see Figure 1 below for $N=5$.

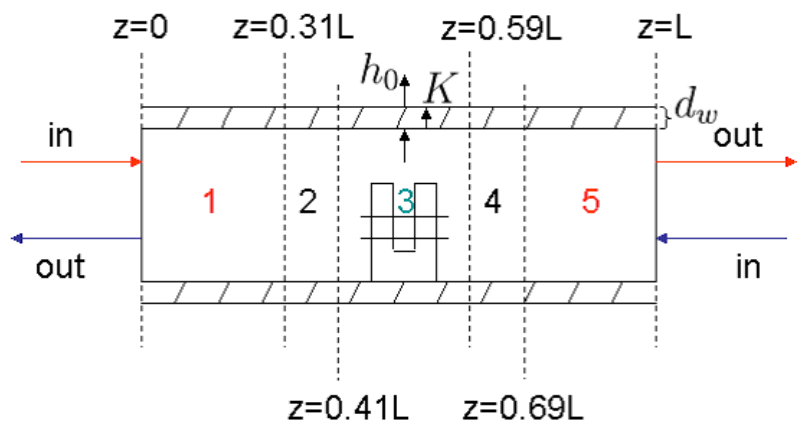

Figure 1. A simplified picture of RFR with switching time and compartments. 1 and 5 are inlet parts, 2 and 4 are catalyst parts, and 3 is a heat exchanger part. In this case, $L_{1}=0.31 L, L_{2}=0.41 L, L_{3}=0.59 L$ and $L_{4}=0.69 L$.

For simplicity, we focus on temperature of the gas and the solid. Then, the equation of the $j$ th room $(i=1,2, \ldots, N-1, N)$ can be described by (4.1) in $\left(L_{j-1}, L_{j}\right) \times(0, T)\left(0=L_{0}<L_{1}<L_{2}<\cdots<L_{N-1}<L_{N}=L\right)$, and we denote the unknown functions by $\left(\Theta_{s}^{(j)}, \Theta_{g}^{(j)}, X^{(j)}\right)$. The initial condition is imposed as follows:

$$
\left.\Theta_{s}^{(j)}\right|_{t=0}=\Theta_{s, 0},\left.\quad \Theta_{g}^{(j)}\right|_{t=0}=\Theta_{g, 0},\left.\quad X^{(j)}\right|_{t=0}=X_{0}, \quad \text { on }\left[L_{j-1}, L_{j}\right],
$$

for $j=1,2, \cdots, N$, where $\Theta_{s, 0}=\Theta_{s, 0}(x), \Theta_{g, 0}=\Theta_{g, 0}(x), X_{0}=X_{0}(x)$ are given $C^{1}$ functions on $[0, L]$. The boundary condition is imposed as follows:

$$
\begin{array}{lll}
\Theta_{g}=\frac{1-\kappa(t)}{2} \Theta_{g}, & \Theta_{s}=\frac{1-\kappa(t)}{2} X, & \text { at }\{0\} \times(0, T), \\
\Theta_{g}=\frac{1+\kappa(t)}{2} \Theta_{g}, & \Theta_{s}=\frac{1-\kappa(t)}{2} X, & \text { at }\{L\} \times(0, T),
\end{array}
$$

where $\kappa(t)$ is as in (1.2) and " $\Theta_{g}=\Theta_{g}$ " or " $\Theta_{s}=\Theta_{s}$ " is regarded as an empty condition. Note that any boundary conditions do not imposed on for $X=X(x, t)$ and for $\Theta_{g}$ and $\Theta_{s}$ at $\left\{L_{j}\right\} \times(0, T)(j=1, \ldots, N-1)$.

We now give the existence and uniqueness of solutions global in time to the system stated above in $(\mathrm{A})-(\mathrm{C})$. The proofs are similar to that of Theorem 1 or easier than that of 1 . Therefore, we shall only state the results.

Theorem 2. Assume that $\Theta_{g}, X \in C^{1}(0, L] \cap L^{\infty}(0, L)$ and $\Theta_{s} \in C^{1}[0, T]$, and 
that $W_{1}$ and $W_{2}$ satisfy

$$
W_{1}, W_{2} \in C\left(\mathbf{R}^{2}\right), \quad\left|W_{i}(\xi, \eta)\right| \leq K(1+|\xi|+|\eta|), \quad \text { for any }(\xi, \eta) \in \mathbf{R}^{2},
$$

for $i=1,2$ and for some $K>0$ Then, there exists a unique solution $\left(\Theta_{s}, \Theta_{g}, X\right)$ to (4.1)-(4.4) such that

$$
\begin{aligned}
& \Theta_{g}, X \in C^{1,1}\left((\bar{Q} \backslash \Gamma) \cup \Gamma_{2,0}^{\prime}\right) \cap C\left(\bar{Q} \backslash\left(\Gamma_{2} \cup \Gamma_{3}\right)\right), \\
& \Theta_{s} \in C^{1,1}(Q) \cup L_{l o c}^{\infty}(Q) .
\end{aligned}
$$

\section{Acknowledgements}

The authors wish to express their sincere thanks to Professor Y.W. Budhi for drawing our attentions to the model. This work was partially supported by JSPS Grant-in-Aid for Scientific Research (A) 26247013.

\section{References}

[1] A. Bressan. Hyperbolic systems of conservation laws. The one-dimensional Cauchy Problem. Oxford University Press, Oxford, 2000.

[2] Y.W. Budhi, A. Jaree, J.H.B.J. Hoebink and J.C. Schouten. Simulation of reverse flow operation for manipulation of catalyst survace coverage in the selective oxidation of ammonia. Chemical Engineering Science, 59(19):4125-4135, 2004. http://dx.doi.org/10.1016/j.ces.2004.04.040.

[3] G. Eigenberger and U. Nieken. Catalytic combustion with periodic flow reversal. Chemical Engineering Science, 43(8):2109-2115, 1988. http://dx.doi.org/10.1016/0009-2509(88)87091-X.

[4] R. Quinta Ferreira, C. Almeida Costa and S. Masetti. Reverse-flow reactor for selective oxidation process. Chemical Engineering Science, 54(20):4615-4627, 1999. http://dx.doi.org/10.1016/S0009-2509(99)00161-X.

[5] A. Friedman. Partial differential equations of parabolic type. Prentice-Hall, Englewood Cliffs, N.J., 1964.

[6] R. Garg, A. Garayhi and D. Luss. Influence of product adsorption on the operation of reverse-flow reactor. AIChE Journal, 48(2):334-344, 2002. http://dx.doi.org/10.1002/aic.690480217.

[7] V.K. Gupta and S.K. Bhatia. Solution of cyclic profiles in catalytic reactor operation with periodic flow reversal. Computers Chem. Eng., 15(4):229-237, 1991. http://dx.doi.org/10.1016/0098-1354(91)85010-R.

[8] R.E. Hayes. Catalytic solutions for fugitive methane emissions in the oil and gas sector. Chemical Engineering Science, 59(19):4073-4080, 2004. http://dx.doi.org/10.1016/j.ces.2004.04.038.

[9] T.N. Haynes, Ch. Georgakis and H.S. Caram. The application of reverse flow reactors to endothermic reactions. Chemical Engineering Science, 47(9):29272932, 1992. http://dx.doi.org/10.1016/0009-2509(92)87153-H. 
[10] K.G.W. Hung, D. Papadias, P. Björnbom, M. Anderlund and B. Åkermark. Reverse-flow operation for application of imperfectly immobilized catalysts. AIChE Journal, 49(1):151-167, 2003. http://dx.doi.org/10.1002/aic.690490114.

[11] J. Khinast, A. Gurumoorthy and D. Luss. Complex dynamic features of a cooled reverse-flow reactor. AIChE Journal, 44(5):1128-1140, 1998. http://dx.doi.org/10.1002/aic.690440511.

[12] O.A. Ladyzhenskaja, V.A. Solonnikov and N.N. Uraceva. Linear and quasilinear equations of parabolic type (in Russian). Nauka, Moscow; English Translation: Transl. Math. Monographs Vol. 23, AMS, Providence, Rhode Island 1968, 1967.

[13] G.M. Lieberman. Second order parabolic differential equations. World Scientific Publishing Co., River Edge, NJ, 1996. http://dx.doi.org/10.1142/3302.

[14] Y.Sh. Matros. Unsteady processes in catalytic reactors. Elsevier, Amsterdam, 1985.

[15] Y.Sh. Matros. Catalytic processes under unsteady-state conditions. Elsevier, Amsterdam, 1989.

[16] Y.Sh. Matros and G.A. Bunimovich. Reverse-flow operation in fixed bed catalytic reactors. Catalysis Reviews. Science and Engineering, 38:1-68, 1996.

[17] V.M. Zahn, M. Mangold, M. Krasnyk and A. Seidel-Morgenstern. Theoretical analysis of heat integration in a periodically operated canscade of catalytic fixed-bed reactors. Chemical Engineering \& Science, 32(9):1326-1338, 2009. http://dx.doi.org/10.1002/ceat.200900201.

\section{Appendix}

\section{Variables:}

$x$ : space variable (axial coordinate),

$t$ : time variable.

(Unknown) Functions:

$\Theta=\Theta(x, t)$ : temperature of the catalyst,

$X=X(x, t)$ : fractional conversion of the catalyst,

$X_{s}=X_{s}(x, t)$ : fractional conversion on the surface of the catalyst pellet,

$X_{g}=X_{g}(x, t)$ : conversion in the gas phase.

(Given Positive) Constants:

$L$ : bed length,

$\varepsilon_{g}$ : fraction of gas phase in packed bed,

$\rho_{g}$ : gas-phase density,

$c_{g}:$ gas-phase heat capacity,

$\varepsilon_{s}$ : fraction of solid phase in packed bed,

$\rho_{s}$ : solid-phase density,

$c_{s}$ : solid-phase heat capacity,

$\lambda_{e f}$ : effective heat conductivity,

$\lambda_{e f, s}$ : effective heat conductivity of packed bed solid phase,

$\rho_{g}^{o}$ : gas-phase density at normal temperature and pressure,

$-\delta H$ : heat of reaction,

$\Theta_{\text {inp }}$ : inlet temperature,

$\beta_{0}$ : mass transfer coefficient, 
$\eta$ : effectiveness factor,

$t_{c}$ : space time,

$n$ : number of switches.

(Given) Functions: $U(t)=\kappa(t) u$ : linear velocity,

$u$ : absolute value of linear velocity

$$
\kappa(t)= \begin{cases}+1, & \text { at } t \in\left[2 m T_{*},(2 m+1) T_{*}\right), \\ -1, & \text { at } t \in\left[(2 m+1) T_{*},(2 m+2) T_{*}\right),\end{cases}
$$

for $m=0,1, \ldots$.

$\tau^{*}$ : half of the cycle duration,

$W_{1}=W_{1}(X, \Theta)$ : rate of chemical reaction,

$W_{2}=W_{2}(X, \Theta)$ : rate of chemical reaction.

Notations: $Q_{T}=(0, L) \times(0, T)$,

$\overline{Q_{T}}=$ closure of $Q_{T}$,

$\left(\Omega \subset \mathbf{R}^{d}\right)$ : open set,

$\operatorname{Lip}(\Omega)$ : Space of continuous functions on $\Omega$ which is satisfy Lipschitz condition, i.e. $0<\gamma \leq 1, u: \Omega \rightarrow \mathbf{R}$ satisfy the estimate

$|u(x)-u(y)| \leq{ }^{\exists} C|x-y|(\forall x, y \in \Omega)$,

$C^{k}(\Omega)$ : space of continuous functions possessing continuous derivatives up to order $k$ on $\Omega$,

$C^{k}(\bar{\Omega})$ : space of all $u \in C^{k}(\Omega)$ such that $u$ and $\partial^{\alpha} u(|u| \leq k)$ extend continuously to the closure $\bar{\Omega}$, where $\alpha$ is a multiindex,

$C^{k, \gamma}(\bar{\Omega})$ : Hölder spaces,i.e. space of all functions $u \in C^{k}(\bar{\Omega})$ and whose $k^{t h}$ -partial derivatives are Hölder continuous with exponent $\gamma$.

In other words

$$
C^{k, \gamma}(\bar{\Omega}):=\left\{u \in C^{k}(\bar{\Omega}) \mid\|u\|_{C^{k, \gamma}(\bar{\Omega})}<\infty\right\}
$$

with

$$
\|u\|_{C^{k, \gamma}(\bar{\Omega})}:=\sum_{|\alpha| \leq k}\left\|\partial^{\alpha} u\right\|_{C(\bar{\Omega})}+\sum_{|\alpha|=k}\left[\partial^{\alpha} u\right]_{C^{0, \gamma}(\bar{\Omega})},
$$

where

$$
[u]_{C^{0, \gamma}(\bar{\Omega})}:=\sup _{x, y \in \Omega, x \neq y}\left\{\frac{|u(x)-u(y)|}{|x-y|^{\gamma}}\right\} .
$$

